\title{
Lyapunov-type inequality and solution for a fractional differential equation
}

Dexiang $\mathrm{Ma}^{1 *}$ and Zifa Yang ${ }^{1}$

"Correspondence: mdxcxg@163.com

'Department of Mathematics, North China Electric Power University, Beijing, 102206, China

\section{Abstract}

In this paper, we consider the linear fractional differential equation

$$
\left\{\begin{array}{l}
{ }_{0}^{C} \mathscr{D}_{t}^{v} u(t)+q(t) u(t)=0, \quad t \in(0,1), v \in(1,2], \\
u(0)=\delta u(1), \quad u^{\prime}(0)=\gamma u^{\prime}(1) .
\end{array}\right.
$$

By obtaining the Green's function we derive the Lyapunov-type inequality for such a boundary value problem. Furthermore, we use the contraction mapping theorem to study the existence of a unique solution for the corresponding nonlinear problem.

MSC: $34 \mathrm{~A} 08$

Keywords: Fractional differential equation; Green's function; Lyapunov-type inequality; Contraction mapping theorem

\section{Introduction}

In 1907, Lyapunov [1] stated the following outstanding result.

Theorem 1.1 ([1]) If the boundary value problem (BVP)

$$
\left\{\begin{array}{l}
y^{\prime \prime}(t)+q(t) y(t)=0, \quad a<t<b \\
y(a)=y(b)=0
\end{array}\right.
$$

has a nontrivial solution, then we have the following Lyapunov inequality:

$$
\int_{a}^{b}|q(s)| d s>\frac{4}{b-a}
$$

Inequality (1) is very useful in various problems related to differential equations. Since the appearance of Lyapunov's fundamental paper [1], many improvements and generalizations of inequality (1) for integer-order (second- and higher-order) BVPs have appeared in the literature; we refer the reader to the summary reference by Tiryaki [2].

Recently, the studies on Lyapunov's inequality for fractional boundary value problem (FBVP) have begun, in which fractional derivatives (Riemann-Liouville derivative ${ }_{a}^{R} \mathscr{D}_{t}^{v}$ or

(c) The Author(s) 2020. This article is licensed under a Creative Commons Attribution 4.0 International License, which permits use, sharing, adaptation, distribution and reproduction in any medium or format, as long as you give appropriate credit to the original author(s) and the source, provide a link to the Creative Commons licence, and indicate if changes were made. The images or other third party material in this article are included in the article's Creative Commons licence, unless indicated otherwise in a credit line to the material. If material is not included in the article's Creative Commons licence and your intended use is not permitted by statutory regulation or exceeds the permitted use, you will need to obtain permission directly from the copyright holder. To view a copy of this licence, visit http://creativecommons.org/licenses/by/4.0/ 
Caputo derivative ${ }_{a}^{C} \mathscr{D}_{t}^{v}$ ) are used instead of the classical ordinary derivative. Such a work was initiated by Ferreira [3] in 2013, who obtained a Lyapunov inequality for the following differential equation with Riemann-Liouville fractional derivative:

$$
\left({ }_{a}^{R} \mathscr{D}_{t}^{v} y\right)(t)+q(t) y(t)=0, \quad a<t<b, 1<v \leq 2,
$$

subject to the boundary value condition

$$
y(a)=y(b)=0 .
$$

Next, in 2014, Ferreira [4] obtained a Lyapunov inequality for the following differential equation with Caputo fractional derivative:

$$
\left({ }_{a}^{C} \mathscr{D}_{t}^{v} y\right)(t)+q(t) y(t)=0, \quad a<t<b, 1<v \leq 2,
$$

subject to boundary value condition (3).

After [3] and [4], many results appeared in the literature; we refer the reader to [510], where Lyapunov or Lyapunov-type inequalities are obtained for fractional differential equation subject various boundary value conditions such as

$$
\begin{aligned}
& y^{\prime}(a)=y^{\prime}(b)=y(c)=0, \quad a<b, c \in[a, b] ; \\
& y(a)=y^{\prime}(a)=0, \quad y^{\prime}(b)=\beta y^{\prime}(\xi) ; \\
& y(a)=y^{\prime}(a)=y^{\prime \prime}(a)=y^{\prime \prime}(b)=0 ; \\
& y(a)=y^{\prime}(a)=y(b)=0 .
\end{aligned}
$$

Inspired by the works mentioned, in this paper, we aim to investigate the Lyapunov-type inequality for the following fractional differential equations:

$$
\left\{\begin{array}{l}
{ }_{0}^{C} \mathscr{D}_{t}^{v} u(t)+q(t) u(t)=0, \quad t \in(0,1), v \in(1,2], \\
u(0)=\delta u(1), \quad u^{\prime}(0)=\gamma u^{\prime}(1),
\end{array}\right.
$$

where $\delta$ and $\gamma$ are real numbers, and $q(t) \in L(0,1)$ is not identically zero on any compact subinterval of $(0,1)$. Furthermore, we obtain the existence of a solution for the corresponding nonlinear problem:

$$
\left\{\begin{array}{l}
{ }_{0}^{C} \mathscr{D}_{t}^{v} u(t)+q(t) f(u(t))=0, \quad t \in(0,1), v \in(1,2], \\
u(0)=\delta u(1), \quad u^{\prime}(0)=\gamma u^{\prime}(1) .
\end{array}\right.
$$

BVP (6) was recently studied in [11], but we should point out that only the case of $\delta>1$ and $0<\gamma<1$ was considered in [11]. In this paper, we give a comprehensive discussion on parameters $\delta$ and $\gamma$.

\section{Preliminaries and lemmas}

For convenience, we present some definitions and lemmas from fractional calculus theory in the sense of Riemann-Liouville and Caputo. 
Definition 2.1 ([12]) Let $\Gamma(v)=\int_{0}^{\infty} t^{v-1} e^{-t} d t, v>0$, be the gamma function. Then the Riemann-Liouville fractional integral of order $v$ for $y(t)$ is defined as

$$
\left({ }_{a} \mathscr{I}_{t}^{v} y\right)(t):=\frac{1}{\Gamma(v)} \int_{a}^{t}(t-s)^{\nu-1} y(s) d s, \quad t \in[a, b]
$$

Definition 2.2 ([12]) Let $v>0$ and $n=[v]+1$, where $[v]$ denotes the integer part of a number $v$. Then the Caputo fractional derivative of order $v$ for $y(t)$ is defined as

$$
\left({ }_{a}^{C} \mathscr{D}_{t}^{v} y\right)(t):=\left({ }_{a} \mathscr{I}_{t}^{n-v} y^{(n)}\right)(t)=\frac{1}{\Gamma(n-v)} \int_{a}^{t} \frac{y^{(n)}(s)}{(t-s)^{v+1-n}} d s, \quad t \in[a, b]
$$

By Definitions 2.1 and 2.2 we have

$$
\left({ }_{a} \mathscr{I}_{t}^{v}\left({ }_{a}^{C} \mathscr{D}_{t}^{v} y\right)\right)(t)=y(t)+C_{1}+C_{2} t+\cdots+C_{n} t^{n-1}
$$

Lemma 2.1 A function $u(t)$ is a solution of the boundary value problem (5) if and only if $u(t)$ satisfies

$$
u(t)=\int_{0}^{1} G(t, s) q(s) u(s) d s
$$

where

$$
G(t, s)=\frac{1}{\Gamma(\nu)}\left\{\begin{array}{c}
(1-v) \frac{\delta \gamma(1-t)+\gamma t}{(1-\gamma)(1-\delta)}(1-s)^{\nu-2}-\frac{\delta}{1-\delta}(1-s)^{\nu-1}-(t-s)^{\nu-1} \\
0 \leq s \leq t \leq 1 \\
(1-v) \frac{\delta \gamma(1-t)+\gamma t}{(1-\gamma)(1-\delta)}(1-s)^{\nu-2}-\frac{\delta}{1-\delta}(1-s)^{\nu-1} \\
0 \leq t \leq s \leq 1
\end{array}\right.
$$

Proof Let $u(t)$ be a solution of (5). Then

$$
\left({ }_{0} \mathscr{I}_{t}^{v}\left({ }_{0}^{C} \mathscr{D}_{t}^{v} u\right)\right)(t)+{ }_{0} \mathscr{I}_{t}^{v}(q(t) u(t))=0
$$

By (7) we obtain

$$
u(t)=C_{1}+C_{2} t-\frac{1}{\Gamma(v)} \int_{0}^{t}(t-s)^{\nu-1} q(s) u(s) d s
$$

Considering $u(0)=\delta u(1)$, we have

$$
C_{1}=\delta C_{1}+\delta C_{2}-\frac{\delta}{\Gamma(v)} \int_{0}^{1}(1-s)^{\nu-1} q(s) u(s) d s
$$

considering $u^{\prime}(0)=\gamma u^{\prime}(1)$, we have

$$
C_{2}=\frac{\gamma(v-1)}{\Gamma(v)(\gamma-1)} \int_{0}^{1}(1-s)^{v-2} q(s) u(s) d s
$$


and thus we get

$$
\begin{aligned}
C_{1}= & \frac{\delta \gamma(v-1)}{\Gamma(v)(1-\delta)(\gamma-1)} \int_{0}^{1}(1-s)^{\nu-2} q(s) u(s) d s \\
& -\frac{\delta}{\Gamma(v)(1-\delta)} \int_{0}^{1}(1-s)^{\nu-1} q(s) u(s) d s .
\end{aligned}
$$

Substituting (10) and (11) into (9), we obtain

$$
\begin{aligned}
u(t)= & \frac{\delta \gamma(v-1)}{\Gamma(v)(1-\delta)(\gamma-1)} \int_{0}^{1}(1-s)^{v-2} q(s) u(s) d s \\
& -\frac{\delta}{\Gamma(v)(1-\delta)} \int_{0}^{1}(1-s)^{v-1} q(s) u(s) d s \\
& +\frac{\gamma(v-1) t}{\Gamma(v)(\gamma-1)} \int_{0}^{1}(1-s)^{v-2} q(s) u(s) d s-\frac{1}{\Gamma(v)} \int_{0}^{t}(t-s)^{v-1} q(s) u(s) d s \\
= & \int_{0}^{1} G(t, s) q(s) u(s) d s,
\end{aligned}
$$

where $G(t, s)$ is the Green's function:

$$
G(t, s)=\frac{1}{\Gamma(v)}\left\{\begin{array}{c}
(1-v) \frac{\delta \gamma(1-t)+\gamma t}{(1-\gamma)(1-\delta)}(1-s)^{\nu-2}-\frac{\delta}{1-\delta}(1-s)^{\nu-1}-(t-s)^{\nu-1} \\
0 \leq s \leq t \leq 1 \\
(1-v) \frac{\delta \gamma(1-t)+\gamma t}{(1-\gamma)(1-\delta)}(1-s)^{\nu-2}-\frac{\delta}{1-\delta}(1-s)^{\nu-1} \\
0 \leq t \leq s \leq 1
\end{array}\right.
$$

Lemma 2.2 When $\delta \in(0,1)$ and $\gamma \in(0,1)$, Green's function $G(t, s)$ satisfies the following properties:

(i) $G(t, s) \leq 0,(t, s) \in[0,1] \times[0,1]$;

(ii) $\max _{0 \leq t \leq 1}|G(t, s)|=-G(1, s)=\frac{(1-s)^{\nu-2}}{\Gamma(v)(1-\delta)(1-\gamma)}[\gamma(v-1)+(1-\gamma)(1-s)]$ for $s \in[0,1]$,

(iii) $\int_{0}^{1}|G(t, s)| d s \leq \frac{\gamma(v-1)+1}{\Gamma(v+1)(1-\delta)(1-\gamma)}$.

Proof (i) $G(t, s) \leq 0$ is obvious since $\delta \in(0,1)$ and $\gamma \in(0,1)$.

(ii) For $s \in[0,1]$ and $t \in[s, 1]$, we have

$$
G_{t}^{\prime}(t, s)=\frac{1-v}{\Gamma(\nu)}\left[\frac{\gamma}{1-\gamma}(1-s)^{\nu-2}+(t-s)^{\nu-2}\right] \leq 0,
$$

which means

$$
G(1, s) \leq G(t, s) \leq G(s, s) \leq 0, \quad s \leq t \leq 1
$$

for $t \in[0, s]$, we have

$$
G_{t}^{\prime}(t, s)=\frac{\gamma(1-v)}{\Gamma(v)(1-\gamma)}(1-s)^{\nu-2} \leq 0,
$$

which means

$$
G(s, s) \leq G(t, s) \leq G(0, s) \leq 0, \quad 0 \leq t \leq s
$$


Inequalities (12) and (13) show that, for $s \in[0,1]$,

$$
G(1, s) \leq G(t, s) \leq G(0, s) \leq 0, \quad 0 \leq t \leq 1 .
$$

Therefore, for $s \in[0,1]$,

$$
\begin{aligned}
\max _{0 \leq t \leq 1}|G(t, s)| & =-G(1, s) \\
& =\frac{1}{\Gamma(\nu)}\left[\frac{(\nu-1) \gamma}{(1-\delta)(1-\gamma)}(1-s)^{\nu-2}+\frac{(1-s)^{\nu-1}}{1-\delta}\right] \\
& =\frac{(1-s)^{\nu-2}}{\Gamma(\nu)(1-\delta)(1-\gamma)}[\gamma(\nu-1)+(1-\gamma)(1-s)] .
\end{aligned}
$$

(iii) By (ii) we have

$$
\begin{aligned}
\int_{0}^{1}|G(t, s)| d s & \leq \int_{0}^{1}-G(1, s) d s \\
& =\int_{0}^{1} \frac{(1-s)^{\nu-2}}{\Gamma(\nu)(1-\delta)(1-\gamma)}[\gamma(\nu-1)+(1-\gamma)(1-s)] d s \\
& =\frac{\gamma(\nu-1)+1}{\Gamma(\nu+1)(1-\delta)(1-\gamma)}
\end{aligned}
$$

Lemma 2.3 When $\delta \in(1,+\infty)$ and $\gamma \in(0,1)$, Green's function $G(t, s)$ satisfies the following properties:

(i) $G(t, s) \geq 0,(t, s) \in[0,1] \times[0,1]$;

(ii) $\max _{0 \leq t \leq 1}|G(t, s)|=G(0, s)=\frac{\delta(1-s)^{\nu-2}}{\Gamma(v)(\delta-1)(1-\gamma)}[\gamma(\nu-1)+(1-\gamma)(1-s)]$ for $s \in[0,1]$,

(iii) $\int_{0}^{1}|G(t, s)| d s \leq \frac{\delta(\gamma v+1-\gamma)}{\Gamma(v+1)(\delta-1)(1-\gamma)}$.

Proof (i) When $0 \leq t \leq s \leq 1$,

$$
G(t, s)=(\nu-1) \frac{\delta \gamma(1-t)+\gamma t}{\Gamma(\nu)(\delta-1)(1-\gamma)}(1-s)^{\nu-2}+\frac{\delta}{\Gamma(\nu)(\delta-1)}(1-s)^{\nu-1} \geq 0
$$

When $0 \leq s \leq t \leq 1$,

$$
\begin{aligned}
G(t, s) & =(\nu-1) \frac{\delta \gamma(1-t)+\gamma t}{\Gamma(\nu)(\delta-1)(1-\gamma)}(1-s)^{\nu-2}+\frac{(1-s)^{\nu-1}}{\Gamma(\nu)}\left[\frac{1}{\delta-1}+1-\left(\frac{t-s}{1-s}\right)^{\nu-1}\right] \\
& \geq 0
\end{aligned}
$$

(ii) For $s \in[0,1]$ and $t \in[s, 1]$, we have

$$
G_{t}^{\prime}(t, s)=\frac{1-v}{\Gamma(\nu)}\left[\frac{\gamma}{1-\gamma}(1-s)^{\nu-2}+(t-s)^{\nu-2}\right] \leq 0,
$$

which means

$$
0 \leq G(1, s) \leq G(t, s) \leq G(s, s), \quad s \leq t \leq 1
$$


For $t \in[0, s]$, we have

$$
G_{t}^{\prime}(t, s)=\frac{\gamma(1-v)(1-s)^{\nu-2}}{(1-\gamma) \Gamma(\nu)} \leq 0
$$

which means

$$
G(s, s) \leq G(t, s) \leq G(0, s), \quad 0 \leq t \leq s .
$$

Inequalities (14) and (15) show us that, for $s \in[0,1]$,

$$
\max _{0 \leq t \leq 1}|G(t, s)|=G(0, s)=\frac{\delta(1-s)^{\nu-2}}{\Gamma(\nu)(\delta-1)(1-\gamma)}[\gamma(\nu-1)+(1-\gamma)(1-s)] .
$$

(iii) From (ii) we have

$$
\begin{aligned}
\int_{0}^{1}|G(t, s)| d s & \leq \int_{0}^{1} G(0, s) d s \\
& =\int_{0}^{1} \frac{\delta(1-s)^{\nu-2}}{\Gamma(\nu)(\delta-1)(1-\gamma)}[\gamma(\nu-1)+(1-\gamma)(1-s)] d s \\
& =\frac{\delta(\gamma \nu+1-\gamma)}{\Gamma(\nu+1)(\delta-1)(1-\gamma)} .
\end{aligned}
$$

Lemma 2.4 When $\delta \in(0,1)$ and $\gamma \in\left(1,1+\frac{(v-1) \delta}{2-v}\right]$, Green's function $G(t, s)$ satisfies the following properties:

(i) $G(t, s) \geq 0,(t, s) \in[0,1] \times[0,1]$;

(ii) $\max _{0 \leq t \leq 1}|G(t, s)|=G(1, s)=\frac{(1-s)^{\nu-2}}{\Gamma(v)(1-\delta)(\gamma-1)}[\gamma(v-1)-(\gamma-1)(1-s)]$ for $s \in[0,1]$,

(iii) $\int_{0}^{1}|G(t, s)| d s \leq \frac{\gamma(v-1)+1}{\Gamma(v+1)(1-\delta)(\gamma-1)}$.

Proof We first prove (i) and (ii). For $s \in[0,1]$, when $t \in[0, s]$,

$$
G_{t}^{\prime}(t, s)=\frac{\gamma(\nu-1)(1-s)^{\nu-2}}{\Gamma(\nu)(\gamma-1)} \geq 0
$$

which means that, for $s \in[0,1]$,

$$
G(0, s) \leq G(t, s) \leq G(s, s), \quad t \in[0, s]
$$

When $t \in[s, 1]$,

$$
\begin{aligned}
& G_{t}^{\prime}(t, s)=\frac{(v-1)}{\Gamma(\nu)}\left[\frac{\gamma}{\gamma-1}(1-s)^{\nu-2}-(t-s)^{\nu-2}\right], \\
& G_{t t}^{\prime \prime}(t, s)=\frac{1}{\Gamma(\nu)}(v-1)(2-v)(t-s)^{\nu-3} \geq 0 .
\end{aligned}
$$

Letting $G_{t}^{\prime}(t, s)=0$, we get $t^{*}=\left(\frac{\gamma}{\gamma-1}\right)^{\frac{1}{v-2}}(1-s)+s \in[s, 1]$. Combining with (17), for $s \in$ $[0,1]$, we have

$$
\begin{array}{ll}
G\left(t^{*}, s\right) \leq G(t, s) \leq G(s, s), & t \in\left[s, t^{*}\right], \\
G\left(t^{*}, s\right) \leq G(t, s) \leq G(1, s), & t \in\left[t^{*}, 1\right] .
\end{array}
$$


Inequalities (16), (18), and (19) show us that, for $s \in[0,1]$,

$$
\max _{0 \leq t \leq 1}|G(t, s)|=\max \left\{|G(0, s)|,|G(s, s)|,\left|G\left(t^{*}, s\right)\right|,|G(1, s)|\right\} .
$$

Now we prove $G(0, s), G(s, s), G\left(t^{*}, s\right)$, and $G(1, s)$ are all nonnegative.

For $G(0, s)$, we have

$$
\begin{aligned}
& G(0, s)=\frac{1}{\Gamma(\nu)}\left[\frac{\delta \gamma(v-1)}{(1-\delta)(\gamma-1)}(1-s)^{\nu-2}-\frac{\delta}{1-\delta}(1-s)^{\nu-1}\right], \\
& G_{s}^{\prime}(0, s)=\frac{\delta(\nu-1)(1-s)^{\nu-3}}{(1-\delta) \Gamma(\nu)}\left[(1-s)+\frac{\gamma(2-v)}{\gamma-1}\right] \geq 0,
\end{aligned}
$$

which means that $G(0, s)$ is increasing for $s \in[0,1]$. Considering that $\gamma<\frac{1}{2-v}$ in case of $1<\gamma \leq 1+\frac{(\nu-1) \delta}{2-v}$ and $0<\delta<1$, we have, for $s \in[0,1]$,

$$
G(0, s) \geq G(0,0)=\frac{\delta}{\Gamma(\nu)(1-\delta)(\gamma-1)}[1-\gamma(2-v)]>0
$$

Inequalities(16) and (20) show that $G(s, s)>0$.

For $G\left(t^{*}, s\right)$, we have

$$
\begin{aligned}
G\left(t^{*}, s\right)= & \frac{(1-s)^{\nu-2}}{\Gamma(\nu)}\left\{\frac{\delta \gamma(\nu-1)}{(\gamma-1)(1-\delta)}+\left[(2-v)\left(\frac{\gamma}{\gamma-1}\right)^{\frac{\nu-1}{\nu-2}}+\frac{\delta}{1-\delta}\right](s-1)\right. \\
& \left.+\frac{\gamma(v-1)}{\gamma-1} s\right\} \\
= & \frac{(1-s)^{\nu-2}}{\Gamma(\nu)} g(s),
\end{aligned}
$$

where

$$
g(s)=\frac{\delta \gamma(v-1)}{(\gamma-1)(1-\delta)}+\left[(2-v)\left(\frac{\gamma}{\gamma-1}\right)^{\frac{\nu-1}{\nu-2}}+\frac{\delta}{1-\delta}\right](s-1)+\frac{\gamma(v-1)}{\gamma-1} s, \quad s \in[0,1] .
$$

Obviously, $g(s)$ is increasing on $[0,1]$, and thus

$$
g(s) \geq g(0)=\frac{\delta \gamma(\nu-1)}{(\gamma-1)(1-\delta)}-(2-v)\left(\frac{\gamma}{\gamma-1}\right)^{\frac{\nu-1}{\nu-2}}-\frac{\delta}{1-\delta}, \quad s \in[0,1]
$$

Let $k(t)=\frac{\delta(v-1)}{1-\delta} t-(2-v) t^{\frac{v-1}{v-2}}-\frac{\delta}{1-\delta}, t \in[1,+\infty)$. Then

$$
k^{\prime}(t)=\frac{\delta(v-1)}{1-\delta}+(v-1) t^{\frac{1}{v-2}} \geq 0
$$

which mean that $k(t)$ is increasing in $[1,+\infty)$. Letting $k\left(t_{0}\right)=0$, we get $t_{0}=\frac{(1-\delta)(2-v) t_{0}^{\frac{\nu-1}{\nu-2}}+\delta}{\delta(v-1)}$ and

$$
t_{0}-1=\frac{(1-\delta)(2-v) t_{0}^{\frac{\nu-1}{\nu-2}}+\delta(2-v)}{\delta(\nu-1)}>0
$$


Then

$$
\begin{aligned}
\frac{t_{0}}{t_{0}-1} & =1+\frac{1}{t_{0}-1} \\
& =1+\frac{(v-1) \delta}{\delta(2-v)+(1-\delta)(2-v) t_{0}^{\frac{v-1}{\nu-2}}} \\
& >1+\frac{(v-1) \delta}{2-v} \geq \gamma,
\end{aligned}
$$

that is, $t_{0}<\frac{\gamma}{\gamma-1}$. By (23) we obtain $g(0)=k\left(\frac{\gamma}{\gamma-1}\right) \geq k\left(t_{0}\right)=0$, and thus

$$
g(s) \geq g(0) \geq 0
$$

From (21) and (24) it follows that $G\left(t^{*}, s\right) \geq 0$.

Since $G\left(t^{*}, s\right) \geq 0$, by (19) it follows that $G(1, s) \geq 0$

Above all, we conclude that

$$
G(t, s) \geq 0, \quad(t, s) \in[0,1] \times[0,1]
$$

and

$$
\max _{0 \leq t \leq 1}|G(t, s)|=\max \{G(s, s), G(1, s)\}
$$

Since $\gamma<\frac{1}{2-\nu}$ in the case of $1<\gamma \leq 1+\frac{(\nu-1) \delta}{2-\nu}$ and $0<\delta<1$, we get

$$
\begin{aligned}
G(s, s)-G(1, s) & =\frac{(1-v)(1-s)^{\nu-2}}{\Gamma(\nu)}\left[\frac{\delta \gamma(1-s)+\gamma s}{(1-\gamma)(1-\delta)}-\frac{\gamma}{(1-\gamma)(1-\delta)}+(1-s)^{\nu-1}\right] \\
& =\frac{(1-s)^{\nu-1}}{\Gamma(\nu)(\gamma-1)}[(2-\nu) \gamma-1] \leq 0,
\end{aligned}
$$

So

$$
\max _{0 \leq t \leq 1}|G(t, s)|=G(1, s)=\frac{(1-s)^{\nu-2}}{\Gamma(\nu)(1-\delta)(\gamma-1)}[\gamma(\nu-1)-(\gamma-1)(1-s)]
$$

(iii) By (ii) we have

$$
\begin{aligned}
\int_{0}^{1}|G(t, s)| d s & \leq \int_{0}^{1} G(1, s) d s \\
& =\int_{0}^{1} \frac{(1-s)^{\nu-2}}{\Gamma(\nu)(1-\delta)(\gamma-1)}[\gamma(\nu-1)-(\gamma-1)(1-s)] d s \\
& =\frac{\gamma(\nu-1)+1}{\Gamma(\nu+1)(1-\delta)(\gamma-1)}
\end{aligned}
$$

Lemma 2.5 When $\delta \in(1,+\infty)$ and $\gamma \in\left(1, \frac{1}{2-\nu}\right]$, Green's function $G(t, s)$ satisfies the following properties:

(i) $G(t, s) \leq 0,(t, s) \in[0,1] \times[0,1]$; 
(ii) For $s \in[0,1]$,

$$
\begin{aligned}
\max _{0 \leq t \leq 1}|G(t, s)| & \\
\leq & \frac{(1-s)^{\nu-2}}{\Gamma(v)(\delta-1)(\gamma-1)} \\
& \times\left\{\delta \gamma(\nu-1)-\left[\delta(\gamma-1)-\gamma(2-v)(\delta-1)\left(\frac{\gamma-1}{\gamma}\right)^{\frac{1}{2-\nu}}\right](1-s)\right\} ;
\end{aligned}
$$

(iii) $\int_{0}^{1}|G(t, s)| d s \leq \frac{1}{\Gamma(v+1)(\delta-1)(\gamma-1)}\left\{\delta[1+\gamma(v-1)]+(\delta-1) \gamma(2-v)\left(\frac{\gamma-1}{\gamma}\right)^{\frac{1}{2-\nu}}\right\}$.

Proof We first prove (i) and (ii). For $s \in[0,1]$ and $t \in[0, s]$,

$$
G_{t}^{\prime}(t, s)=\frac{\gamma(\nu-1)(1-s)^{\nu-2}}{(\gamma-1) \Gamma(v)} \geq 0
$$

which means

$$
G(0, s) \leq G(t, s) \leq G(s, s), \quad t \in[0, s]
$$

When $t \in[s, 1]$,

$$
\begin{aligned}
& G_{t}^{\prime}(t, s)=\frac{(v-1)}{\Gamma(v)}\left[\frac{\gamma}{\gamma-1}(1-s)^{\nu-2}-(t-s)^{v-2}\right], \\
& G_{t t}^{\prime \prime}(t, s)=\frac{1}{\Gamma(v)}(v-1)(2-v)(t-s)^{\nu-3} \geq 0 .
\end{aligned}
$$

Letting $G_{t}^{\prime}(t, s)=0$, we get $t^{*}=\left(\frac{\gamma}{\gamma-1}\right) \frac{1}{v-2}(1-s)+s \in[s, 1]$. Combining (26), for $s \in[0,1]$, we have

$$
\begin{array}{ll}
G\left(t^{*}, s\right) \leq G(t, s) \leq G(s, s), \quad t \in\left[s, t^{*}\right], \\
G\left(t^{*}, s\right) \leq G(t, s) \leq G(1, s), \quad t \in\left[t^{*}, 1\right] .
\end{array}
$$

Inequalities (25), (27), and (28) show that, for $s \in[0,1]$,

$$
\max _{0 \leq t \leq 1}|G(t, s)|=\max \left\{|G(0, s)|,|G(s, s)|,\left|G\left(t^{*}, s\right)\right|,|G(1, s)|\right\} .
$$

We now prove that $G(0, s), G(s, s), G\left(t^{*}, s\right), G(1, s)$ are all nonpositive.

For $G(s, s)$, we have

$$
\begin{aligned}
G(s, s) & =\frac{(1-s)^{\nu-2}}{\Gamma(v)(\delta-1)(\gamma-1)}\{[(2-v) \gamma-1] \delta+[(\nu-1) \delta \gamma-(\gamma-1) \delta-(v-1) \gamma] s\} \\
& =\frac{(1-s)^{\nu-2}}{\Gamma(v)(\delta-1)(\gamma-1)} L(s),
\end{aligned}
$$

where

$$
L(s)=[(2-v) \gamma-1] \delta+[(v-1) \delta \gamma-(\gamma-1) \delta-(v-1) \gamma] s, \quad s \in[0,1] .
$$


We have

$$
L(0)=\delta[(2-v) \gamma-1] \leq 0, \quad L(1)=-(v-1) \gamma<0,
$$

which means that $L(s) \leq 0, s \in[0,1]$, and therefore

$$
G(s, s) \leq 0, \quad s \in[0,1]
$$

Inequalities $G(0, s) \leq 0$ and $G\left(t^{*}, s\right) \leq 0$ follow from (25), (27), and $G(s, s) \leq 0$.

For $G(1, s)$, we have

$$
\begin{aligned}
& G(1, s)=\frac{1}{\Gamma(\nu)}\left[\frac{\gamma(\nu-1)}{(1-\delta)(\gamma-1)}(1-s)^{\nu-2}-\frac{1}{1-\delta}(1-s)^{\nu-1}\right], \\
& G_{s}^{\prime}(1, s)=\frac{-(\nu-1)(1-s)^{\nu-3}}{\Gamma(\nu)(\delta-1)(\gamma-1)}[(2-v) \gamma+(\gamma-1)(1-s)] \leq 0,
\end{aligned}
$$

and thus, for $s \in[0,1]$

$$
G(1, s) \leq G(1,0)=\frac{1}{\Gamma(\nu)(\delta-1)(\gamma-1)}[\gamma(2-v)-1] \leq 0 .
$$

Above all, we get that $G(t, s) \leq 0$ and, for $s \in[0,1]$,

$$
\max _{0 \leq t \leq 1}|G(t, s)|=\max \left\{-G(0, s),-G\left(t^{*}, s\right)\right\} .
$$

We can easily compute that

$$
-G\left(t^{*}, s\right)=\frac{(1-s)^{\nu-2}}{\Gamma(\nu)} h_{1}(s),
$$

where

$$
\begin{aligned}
h_{1}(s)= & \left\{\frac{\delta[1-\gamma(2-v)]}{(\delta-1)(\gamma-1)}+(2-v)\left(\frac{\gamma}{\gamma-1}\right)^{\frac{v-1}{v-2}}\right\} \\
& -\left\{\frac{\delta[1-\gamma(2-v)]}{(\delta-1)(\gamma-1)}+(2-v)\left(\frac{\gamma}{\gamma-1}\right)^{\frac{v-1}{v-2}}-\frac{\gamma(\nu-1)}{(\delta-1)(\gamma-1)}\right\} s,
\end{aligned}
$$

and

$$
-G(0, s)=\frac{(1-s)^{v-2}}{\Gamma(v)} h_{2}(s)
$$

where

$$
h_{2}(s)=\frac{\delta[1-\gamma(2-v)]}{(\delta-1)(\gamma-1)}+\frac{\delta}{\delta-1} s \text {. }
$$


Obviously,

$$
\begin{aligned}
& h_{1}(0)=\frac{\delta[1-\gamma(2-v)]}{(\delta-1)(\gamma-1)}+(2-v)\left(\frac{\gamma}{\gamma-1}\right)^{\frac{\nu-1}{v-2}}>\frac{\delta[1-\gamma(2-v)]}{(\delta-1)(\gamma-1)}=h_{2}(0)>0, \\
& h_{2}(1)=\frac{\delta \gamma(\nu-1)}{(\delta-1)(\gamma-1)}>\frac{\gamma(v-1)}{(\delta-1)(\gamma-1)}=h_{1}(1)>0 .
\end{aligned}
$$

So, if we make a line $H(s)$ through $\left(0, h_{1}(0)\right)$ and $\left(1, h_{2}(1)\right)$, that is,

$$
H(s)=\frac{\delta[1-\gamma(2-v)]}{(\delta-1)(\gamma-1)}+(2-v)\left(\frac{\gamma}{\gamma-1}\right)^{\frac{\nu-1}{\nu-2}}+\left[\frac{\delta}{\delta-1}-(2-v)\left(\frac{\gamma}{\gamma-1}\right)^{\frac{v-1}{\nu-2}}\right] s
$$

then we have

$$
0 \leq h_{1}(s), h_{2}(s) \leq H(s), \quad s \in[0,1] .
$$

Therefore, for $s \in[0,1]$,

$$
\begin{aligned}
\max _{0 \leq t \leq 1}|G(t, s)| & \\
\quad= & \max \left\{-G(0, s),-G\left(t^{*}, s\right)\right\} \\
& \left.=\frac{(1-s)^{\nu-2}}{\Gamma(v)} \max \left\{h_{1}(s), h_{2}(s)\right)\right\} \\
& \leq \frac{(1-s)^{\nu-2}}{\Gamma(v)} H(s) \\
& =\frac{(1-s)^{\nu-2}}{\Gamma(\nu)(\delta-1)(\gamma-1)}\left\{\delta \gamma(v-1)-\left[\delta(\gamma-1)-\gamma(2-v)(\delta-1)\left(\frac{\gamma-1}{\gamma}\right)^{\frac{1}{2-\nu}}\right](1-s)\right\} .
\end{aligned}
$$

(iii) easily s follows by (ii):

$$
\begin{aligned}
\int_{0}^{1} & |G(t, s)| d s \\
\leq & \int_{0}^{1} \max _{0 \leq t \leq 1}|G(t, s)| d s \\
\leq & \int_{0}^{1} \frac{(1-s)^{\nu-2}}{\Gamma(\nu)(\delta-1)(\gamma-1)} \\
& \times\left\{\delta \gamma(\nu-1)-\left[\delta(\gamma-1)-\gamma(2-v)(\delta-1)\left(\frac{\gamma-1}{\gamma}\right)^{\frac{1}{2-v}}\right](1-s)\right\} d s \\
= & \frac{1}{\Gamma(\nu+1)(\delta-1)(\gamma-1)}\left\{\delta[1+\gamma(v-1)]+(2-v)(\delta-1) \gamma\left(\frac{\gamma-1}{\gamma}\right)^{\frac{1}{2-\nu}}\right\} .
\end{aligned}
$$

\section{Main result}

Theorem 3.1 Suppose the boundary value problem (5) has a nonzero solution $u(t)$.

(i) If $\delta \in(0,1)$ and $\gamma \in(0,1)$, then

$$
\int_{0}^{1}(1-s)^{\nu-2}[\gamma(v-1)+(1-\gamma)(1-s)]|q(s)| d s>\Gamma(v)(1-\delta)(1-\gamma)
$$


(ii) If $\delta \in(1,+\infty)$ and $\gamma \in(0,1)$, then

$$
\int_{0}^{1}(1-s)^{\nu-2}[\gamma(v-1)+(1-\gamma)(1-s)]|q(s)| d s>\frac{\Gamma(\nu)(\delta-1)(1-\gamma)}{\delta}
$$

(iii) If $\delta \in(0,1)$ and $\gamma \in\left(1,1+\frac{(v-1) \delta}{2-v}\right]$, then

$$
\int_{0}^{1}(1-s)^{\nu-2}[\gamma(\nu-1)-(\gamma-1)(1-s)]|q(s)| d s>\Gamma(\nu)(1-\delta)(\gamma-1)
$$

(iv) if $\delta \in(1,+\infty)$ and $\gamma \in\left(1, \frac{1}{2-\nu}\right]$, then

$$
\begin{aligned}
\int_{0}^{1}(1-s)^{\nu-2}\left\{\delta \gamma(\nu-1)-\left[\delta(\gamma-1)-\gamma(2-v)(\delta-1)\left(\frac{\gamma-1}{\gamma}\right)^{\frac{1}{2-\nu}}\right](1-s)\right\} \\
\quad \times|q(s)| d s \\
>\Gamma(v)(\delta-1)(\gamma-1) .
\end{aligned}
$$

Proof Let $u(t)$ be a nonzero solution of the boundary value problem (5). By Lemma 2.1 we have

$$
u(t)=\int_{0}^{1} G(t, s) q(s) u(s) d s
$$

Let $m=\max _{t \in[0,1]}|u(t)|$. Then

$$
|u(t)| \leq \int_{0}^{1}|G(t, s)||q(s)||u(s)| d s \leq m \int_{0}^{1}|G(t, s)||q(s)| d s .
$$

Next, since $|G(t, s)||q(s)| \leq \max _{0 \leq t \leq 1}|G(t, s)||q(s)|$, but $|G(t, s)||q(s)| \not \equiv \max _{0 \leq t \leq 1}|G(t, s)| \times$ $|q(s)|$, we have

$$
\int_{0}^{1}|G(t, s)||q(s)| d s<\int_{0}^{1} \max _{0 \leq t \leq 1}|G(t, s)||q(s)| d s,
$$

which means

$$
|u(t)|<m \int_{0}^{1} \max _{0 \leq t \leq 1}|G(t, s)||q(s)| d s
$$

that is,

$$
1<\int_{0}^{1} \max _{0 \leq t \leq 1}|G(t, s)||q(s)| d s .
$$

Substituting Lemma 2.2(ii), Lemma 2.3(ii), Lemma 2.4(ii), and Lemma 2.5(ii) into (29), we easily get statements (i), (ii), (iii), and (iv) of Theorem 3.1.

By Theorem 3.1 we have the following conclusions. 


\section{Theorem 3.2}

(i) when $\delta \in(0,1)$ and $\gamma \in(0,1)$, if

$$
\int_{0}^{1}(1-s)^{\nu-2}[\gamma(v-1)+(1-\gamma)(1-s)]|q(s)| d s \leq \Gamma(v)(1-\delta)(1-\gamma),
$$

then the boundary value problem (5) has no nonzero solution.

(ii) when $\delta \in(1,+\infty)$ and $\gamma \in(0,1)$, if

$$
\int_{0}^{1}(1-s)^{\nu-2}[\gamma(v-1)+(1-\gamma)(1-s)]|q(s)| d s \leq \frac{\Gamma(v)(\delta-1)(1-\gamma)}{\delta},
$$

then the boundary value problem (5) has no nonzero solution.

(iii) when $\delta \in(0,1)$ and $\gamma \in\left(1,1+\frac{(\nu-1) \delta}{2-v}\right]$, if

$$
\int_{0}^{1}(1-s)^{\nu-2}[\gamma(v-1)-(\gamma-1)(1-s)]|q(s)| d s \leq \Gamma(v)(1-\delta)(\gamma-1),
$$

then the boundary value problem (5) has no nonzero solution.

(iv) when $\delta \in(1,+\infty)$ and $\gamma \in\left(1, \frac{1}{2-\nu}\right]$, if

$$
\begin{aligned}
& \int_{0}^{1}(1-s)^{\nu-2}\{\delta \gamma(v-1) \\
& \left.\quad-\left[\delta(\gamma-1)-\gamma(2-v)(\delta-1)\left(\frac{\gamma-1}{\gamma}\right)^{\frac{1}{2-\nu}}\right](1-s)\right\}|q(s)| d s \\
& \leq \Gamma(v)(\delta-1)(\gamma-1),
\end{aligned}
$$

then the boundary value problem (5) has no nonzero solution.

Now we consider the existence of solutions to the following nonlinear boundary value problem:

$$
\left\{\begin{array}{l}
{ }_{0}^{C} \mathscr{D}_{t}^{v} u(t)+f(t, u(t))=0, \\
u(0)=\delta u(1), \quad u^{\prime}(0)=\gamma u^{\prime}(1) .
\end{array}\right.
$$

Theorem 3.3 Let $f:[0,1] \times R \rightarrow R$ be continuous and satisfy the following Lipschitz condition with Lipschitz constant $L$ :

$$
\left|f\left(t, u_{1}\right)-f\left(t, u_{2}\right)\right| \leq L\left|u_{1}-u_{2}\right|
$$

for all $\left(t, u_{1}\right),\left(t, u_{2}\right) \in[0,1] \times R$. If

$$
L< \begin{cases}\frac{\Gamma(\nu+1)(1-\delta)(1-\gamma)}{\gamma(\nu-1)+1}, & \delta \in(0,1), \gamma \in(0,1), \\ \frac{\Gamma(\nu+1)(\delta-1)(1-\gamma)}{\delta(\gamma \nu+1-\gamma)}, & \delta \in(1,+\infty), \gamma \in(0,1), \\ \frac{\Gamma(\nu+1)(1-\delta)(\gamma-1)}{\gamma(\nu-1)+1}, & \delta \in(0,1), \gamma \in\left(1,1+\frac{\delta(\nu-1)}{2-\nu}\right], \\ \frac{\Gamma(\nu+1)(\delta-1)(\gamma-1)}{\delta[1+\gamma(\nu-1)]+(\delta-1) \gamma(2-\nu)\left(\frac{\gamma-1}{\gamma}\right) \frac{1}{2-\nu}}, & \delta \in(1,+\infty], \gamma \in\left(1, \frac{1}{2-\nu}\right],\end{cases}
$$

then (30) has a unique solution. 
Proof Let $E$ be the Banach space $C[0,1]$ with norm $\|u\|=\max _{t \in[0,1]}|u(t)|$.

By Lemma 2.1, $u \in E$ is a solution of (30) if and only if it satisfies the integral equation

$$
u(t)=\int_{0}^{1} G(t, s) f(s, u(s)) d s .
$$

Define the operator $T: E \rightarrow E$ by

$$
T u(t)=\int_{0}^{1} G(t, s) f(s, u(s)) d s
$$

Then $T$ is completely continuous. We claim that $T$ has a unique fixed point in $E$. In fact, for any $u_{1}, u_{2} \in E$, we have

$$
\begin{aligned}
\left|T u_{1}(t)-T u_{2}(t)\right| & \leq \int_{0}^{1}|G(t, s)|\left|f\left(s, u_{1}(s)\right)-f\left(s, u_{2}(s)\right)\right| d s \\
& \leq L \int_{0}^{1}|G(t, s)|\left|u_{1}(s)-u_{2}(s)\right| d s \\
& \leq L \int_{0}^{1}|G(t, s)| d s\left\|u_{1}-u_{2}\right\| .
\end{aligned}
$$

Substituting of Lemma 2.2(iii), Lemma 2.3(iii), Lemma 2.4(iii), and Lemma 2.5(iii) into (33), we conclude that $T$ is a contraction mapping and thus obtain the desired result.

\section{Conclusion}

In this paper, we study a linear fractional differential equation. Firstly, by obtaining the Green's function we derive a Lyapunov-type inequality for such a boundary value problem. Furthermore, we use the contraction mapping theorem to study the existence of a unique solution for the corresponding nonlinear problem.

Funding

No funding.

Abbreviations

Not applicable.

Availability of data and materials

Not applicable.

Competing interests

The authors declare that they have no competing interests.

Authors' contributions

The work was finished by the authors' contributions. All authors read and approved the final manuscript.

\section{Publisher's Note}

Springer Nature remains neutral with regard to jurisdictional claims in published maps and institutional affiliations.

Received: 9 October 2019 Accepted: 22 June 2020 Published online: 01 July 2020

\section{References}

1. Lyapunov, A.M.: Problème général de la stabilité du mouvement. Ann. Fac. Sci. Univ. Toulouse 2(2), 203-247 (1907)

2. Tiryaki, A.: Recent developments of Lyapunov-type inequalities. Adv. Dyn. Syst. Appl. 5(2), 231-248 (2010)

3. Ferreira, R.A.C.: A Lyapunov-type inequality for a fractional boundary value problem. Fract. Calc. Appl. Anal. 16(4), 978-984 (2013) 
4. Ferreira, R.A.C.: On a Lyapunov-type inequality and the zeros of a certain Mittag-Leffler function. J. Math. Anal. Appl. 412(2), 1058-1063 (2014)

5. Jleli, M., Samet, B.: Lyapunov-type inequalities for a fractional differential equation with mixed boundary conditions. Math. Inequal. Appl. 18(2), 443-451 (2015)

6. Jleli, M., Ragoub, L., Samet, B.: Lyapunov-type inequality for a fractional differential equation under a Robin boundary conditions. J. Funct. Spaces 2015, 468536 (2015)

7. Rong, J., Bai, C.Z.: Lyapunov-type inequality for a fractional differential equation with fractional boundary conditions. Adv. Differ. Equ. 2015, 82 (2015)

8. Wang, Y.Y., Liang, S.L., Xia, C.X.: A Lyapunov-type inequalities for a fractional differential equation under Sturm-Liouville boundary conditions. Math. Inequal. Appl. 20, 139-148 (2017)

9. O'Regan, D., Samet, B.: Lyapunov-type inequalities for a class of fractional differential equations. J. Inequal. Appl. 2015, 247 (2015)

10. Cabrera, I., Sadarangani, K., Samet, B.: Hartman-Wintner-type inequalities for a class of nonlocal fractional boundary value problems. Math. Methods Appl. Sci. 2016, 1-8 (2016). https://doi.org/10.1002/mma.3972

11. Ma, T., Tian, Y., Huo, Q., Zhang, Y.: Boundary value problem for linear and nonlinear fractional differential equations. Appl. Math. Lett. 2018(86), 1-7 (2018)

12. Kilbas, A.A., Rivero, M., Rodriguez-Germa, L., Trujillo, J.J.: Caputo linear fractional differential equation. IFAC Proc. Vol. 39(11), 52-57 (2010)

\section{Submit your manuscript to a SpringerOpen ${ }^{\circ}$} journal and benefit from:

- Convenient online submission

- Rigorous peer review

- Open access: articles freely available online

- High visibility within the field

- Retaining the copyright to your article

Submit your next manuscript at $\gg$ springeropen.com 